\title{
Fractionnement et caractérisation des lixiviats de centres d'enfouissement technique de déchets ménagers: intérêt de la chromatographie liquide haute performance sur le gel d'exclusion stérique \\ Fractionation and characterization of leachate from a municipal landfill: advantage of high performance size exclusion chromatography
}

\section{F. Le Coupannec et J. J. Peron}

Volume 12, numéro 3, 1999

URI : https://id.erudit.org/iderudit/705364ar

DOI : https://doi.org/10.7202/705364ar

Aller au sommaire du numéro

Éditeur(s)

Université du Québec - INRS-Eau, Terre et Environnement (INRS-ETE)

ISSN

0992-7158 (imprimé)

1718-8598 (numérique)

Découvrir la revue

Citer cet article

Le Coupannec, F. \& Peron, J. J. (1999). Fractionnement et caractérisation des lixiviats de centres d'enfouissement technique de déchets ménagers: intérêt de la chromatographie liquide haute performance sur le gel d'exclusion stérique. Revue des sciences de l'eau / Journal of Water Science, 12(3), 529-543.

https://doi.org/10.7202/705364ar
Résumé de l'article

L'ultrafiltration et la chromatographie d'exclusion stérique haute performance sont utilisées pour la séparation et la caractérisation des composés organiques présents dans les lixiviats de centres d'enfouissement technique de déchets ménagers.

Le fractionnement de la matière organique est obtenu sur des colonnes type TSK PW, en élution eau pH 4 et eau-méthanol. La spectroscopie en UV-visible et en fluorescence, un détecteur évaporatif à diffusion de lumière sont utilisés pour la caractérisation des fractions.

Cette méthode rapide de séparation associée à une multidétection permet une mise en évidence, dans les fractions issues de l'ultrafiltration, de composés organiques caractéristiques. Dans la fraction de poids moléculaires inférieurs à 1000 Daltons, trois familles sont détectées. Les substances humiques et les protéines sont les principaux groupes présents dans la fraction de poids moléculaires supérieurs à 10000 Daltons.
Tous droits réservés @ C Revue des sciences de l'eau, 1999

Ce document est protégé par la loi sur le droit d'auteur. L'utilisation des services d’Érudit (y compris la reproduction) est assujettie à sa politique d'utilisation que vous pouvez consulter en ligne.

https://apropos.erudit.org/fr/usagers/politique-dutilisation/ 


\title{
Fractionnement et caracterisation des lixiviats de centres d'enfouissement technique de déchets ménagers. Intérêt de la chromatographie liquide haute performance sur gel d'exclusion stérique
}

\author{
Fractionation and characterization of leachate \\ from a municipal landfill. \\ Advantage of high performance size exclusion \\ chromatography
}

\author{
F. LE COUPANNEC, D. MORIN et J.J. PERON *
}

Reçu le 28 janvier 1998, accepté le 2 août 1999* .

\section{SUMMARY}

\begin{abstract}
Landfill leachates represent a source of organic pollution characterized by an important organic load, with high chemical oxygen demand in recent sanitary landfills and some organic compounds refractory to biodegradation. Several researchers have examined the organic matter in these landfill leachates. In addition to measuring parameters such as chemical and biological oxygen demands (COD and BOD) and UV-absorbance, different analytical techniques were applied: gas chromatography with flame ionization or mass detection, high performance liquid chromatography, infrared spectroscopy, nuclear magnetic resonance spectrometry, and elementary analysis. Raw leachates or samples after fractionation (on Sephadex gel, by ultrafiltration or by adsorption on XAD resins) were characterized.

Conclusions from these earlier studies were as follows:

- physico-chemical properties of leachates revealed not only a high organic pollution but also diversity and variability according to the age of the sanitary landfill and the climatic conditions;

- gel permeation chromatography and ultrafiltration revealed two main fractions in the leachates: one with molecular weights below 1000 Daltons (Da), and another with molecular weights above 5 000-10 000 Da;

- infrared spectroscopy and nuclear magnetic resonance spectrometry showed functional groups present in the humic and fulvic acid fractions of natural organic matter;

- a varying number of peaks detected by gas chromatography with flame ionization or mass detection proved the complexity of the matrix. Few compounds
\end{abstract}

Laboratoire de Biologie et Chimie moléculaires, Université de Bretagne Sud, Centre de Génie Industriel, Guidel-Plages, 56520 Guidel, France. 
were identified and quantified, with the exception of fatty acids. Moreover this technique was only applicable to molecules with low molecular weight.

The purpose of the present work was to develop a new method of fractionation of organic matter in landfill leachates aiming at studying their characterization and treatment biodegradation. Ultrafiltration, as a prefractionation step, divided the leachate into four fractions according to their molecular weight: above $10000 \mathrm{Da}$, from 10 000-3 000 Da, from 3000-1 000 Da, and below 1000 Da. The second fractionation step was carried out using gel permeation chromatography. This technique has been applied by earlier researchers for the characterization of landfill leachates, but at low pressure on Sephadex gels. In our study, we developed a high performance size-exclusion chromatography method using a polymer based TSK PW column, a hydrophilic cross-linked polyether. Three TSK G3M00 PW columns and one G5000 PW column were tested with water at pH 4 with acetic acid and with a water/methanol mixture as mobile phases. This rapid method of separation, with short retention times, was coupled with on-line multidetection: UV-visible ( $254 \mathrm{~nm}$ - aromatic compounds), fluorescence spectroscopy $(275 / 325 \mathrm{~nm}$, protein-type molecules; $320 / 430 \mathrm{~nm}$, humic-type molecules) and evaporative light scattering detection, ELSD. The ELSD allowed detection of all mineral and organic compounds that did not evaporate at the working temperature $\left(45^{\circ} \mathrm{C}\right)$.

The effect of the sodium chloride concentration on retention times was tested with eluants and columns. Secondary effects, often observed with size-exclusion chromatography, occurred with the gel chosen. The elution of sodium chloride solutions at different concentrations showed that the TSK PW gel bears electronegative charges, and that the density of these charges differs from one column to another. For the leachate we observed this influence: chromatograms obtained on two TSK G3000 columns were different for fractions with molecular weights below $1000 \mathrm{Da}$.

The comparison of chromatograms obtained with the four detection methods provided information about the identity of the types of compounds present. For fractions with molecular weights below $1000 \mathrm{Da}$, separation was performed using a TSK G3000 PW column, with an eluant pH of 4 and a watermethanol mixture; three main families were detected. For fractions with molecular weights above $10000 \mathrm{Da}$, chromatographic separation was improved by elution with water/methanol (70/30) with TSK G5000 and G3000 columns in series; two main groups were identified, humic substances and protein-type compounds. The constituents of the two intermediate fractions with molecular weights between 10000 and $1000 \mathrm{Da}$ were essentially humic substances, identified after separation on a TSK G3000 PW column with water-methanol $(\mathbf{7 0 / 3 0})$ as the eluant.

Key-words: leachate, size-exclusion chromatography, humic substances, fluorescence, evaporative light scattering detection.

\section{RÉSUMÉ}

L'ultrafiltration et la chromatographie d'exclusion stérique haute performance sont utilisées pour la séparation et la caractérisation des composés organiques présents dans les lixiviats de centres d'enfouissement technique de déchets ménagers.

Le fractionnement de la matière organique est obtenu sur des colonnes type TSK PW, en élution eau pH 4 et eau-méthanol. La spectroscopie en UVvisible et en fluorescence, un détecteur évaporatif à diffusion de lumière sont utilisés pour la caractérisation des fractions.

Cette méthode rapide de séparation associée à une multidétection permet une mise en évidence, dans les fractions issues de l'ultrafiltration, de composés 
organiques caractéristiques. Dans la fraction de poids moléculaires inférieurs à 1000 Daltons, trois familles sont détectées. Les substances humiques et les protéines sont les principaux groupes présents dans la fraction de poids moléculaires supérieurs à 10000 Daltons.

Mots clés : lixiviats, chromatographie d'exclusion stérique, substances humiques, fluorescence, détecteur évaporatif à diffusion de lumière.

\section{INTRODUCTION}

La caractérisation chimique des lixiviats générés par les Centres d'Enfouissement Technique (CET) de déchets a été entreprise par de nombreux auteurs. Ainsi, outre la mesure de paramètres globaux tels que $\mathrm{DCO}, \mathrm{DBO}_{5}$, absorbance UV..., des techniques d'identification ou d'analyses plus spécifiques ont été présentées : chromatographie en phase gazeuse avec détection à ionisation de flamme ou détection de masse (FORST et al., 1989 ; HARMSEN, 1993 ; MANNI et al., 1995 ; MEJBRI et al., 1995 ; MERTENS et al., 1995 ; MURRAY et al., 1990 ; OMAN et al., 1993 ; REINHARD et al., 1984 ; SAWHNEY et al., 1984 ; SCHULTZ et al., 1986), chromatographie en phase liquide haute performance (GOURDON et al., 1989), spectroscopie infrarouge, RMN et analyse élémentaire (MEJBRI et al., 1995). Ces caractérisations sont menées sur les effluents bruts ou après fractionnement préalable par extraction liquide-liquide ou par perméation sur gel Sephadex (CHIAN et al., 1977 ; CHAISSAC et al., 1987 ; CLEMENT et al., 1994 ; GOURDON et al., 1989 ; GRANET et al., 1985 ; MILLOT, 1986 ; MILLOT et al., 1987 ; URANO et al., 1980) par ultrafiltration (GOURDON et al., 1989 ; SLATER et al., 1985), par adsorption sur résine Amberlite XAD (MEJBRI et al., 1995). L'examen de ces différents travaux conduit aux conclusions suivantes :

- la chromatographie par perméation sur gel Sephadex et l'ultrafiltration fractionnent la matière organique en deux groupes prédominants de composés: ceux de poids moléculaires apparents (PMA) inférieurs à 1000 Daltons (Da) et ceux de PMA supérieurs à 5 000-10 000 Da ;

- l'exploitation des spectres IR et RMN met en évidence des groupements fonctionnels présents aussi dans les acides humiques et fulviques de la matière organique naturelle ;

- les analyses chromatographiques, essentiellement en phase gazeuse montrent la diversité des composés organiques mais mis à part le dosage des acides gras volatils, peu d'auteurs ont quantifié les composés identifiés.

Ces travaux mettent aussi en évidence les difficultés de caractérisation des lixiviats résultant d'un fractionnement insuffisant de la matière organique. Nous nous sommes donc fixés comme objectif d'améliorer le fractionnement en utilisant la chromatographie d'exclusion stérique haute performance après une préséparation par ultrafiltration ; pour cela, nous avons utilisé des données sur la séparation des substances humiques (DE HAAN et al., 1978 ; DUBIN, 1988 ; HONGVE et al., 1996 ; MATSUBARA et al., 1994 ; POSNER, 1963) et plus généralement sur les macromolécules d'origine naturelle (HEJZLAR, 1987 ; KATO et al., 1980 ; KATO, 1989 ; RINAUDO et al., 1991). 


\section{MATÉRIEL}

\section{L'ultrafiltration tangentielle}

Elle est réalisée sur un appareillage FILTRON, modèle MINISETTE, constitué d'une pompe ULTRAPOMPE $V$ et de cassettes à membrane OMEGA en polyéther sulfone modifié. Trois cassettes de seuil de coupure 10, 3 et 1 kilodaltons (KDa) sont successivement utilisées. Le protocole mis en œuvre donne quatre fractions F1, F2, F3 et F4 (figure 1), de volume voisin de celui du lixiviat brut ultrafiltré.

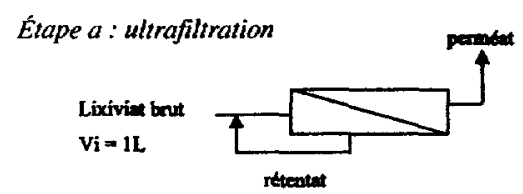

Étape $b$ : rinçage de la membrane - dilution du rétenta

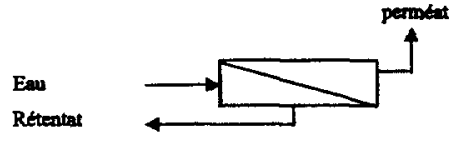

Figure 1 Protocole expérimental de l'ultrafiltration.

Experimental protocol for ultrafiltration.

\section{La chromatographie de perméation de gel}

La chaîne analytique comprend une pompe VARIAN 9012 équipée d'une vanne d'injection VALCO (boucle de $50 \mu \mathrm{l}$ ). Pour la séparation, deux types de colonne TSK Tosohaas $(7.5 \mathrm{~mm} \times 30 \mathrm{~cm})$, sont utilisées : types G3000 PW et G5000 PW (porosité respective des gels : $200 \AA$ et $1000 \AA$ ).

Les éluants sont l'eau acidifiée à $\mathrm{pH} 4$ par de l'acide acétique et le mélange eau-méthanol (Qualité HPLC Carlo Erba) ; le débit est de $0,5 \mathrm{ml} / \mathrm{min}$. Les échantillons sont acidifiés par de l'acide acétique à pH 4, avant leur injection sur la colonne $\mathrm{G} 3000$ en élution eau $\mathrm{pH} 4$.

Trois détecteurs peuvent être couplés à la pompe : un détecteur UV - Visible VARIAN 9050, un détecteur spectrofluorimétrique LaChrom L - 7480 MERCK HITACHI, un détecteur évaporatif à diffusion de lumière (DEDL) SEDEX 55 TOUZART et MATIGNON. Le fonctionnement de ce détecteur, commercialisé depuis une dizaine d'années, est décrit dans l'article de synthèse de DREUX et al. (1996). L'enregistrement des signaux est effectué à l'aide d'un intégrateur SHIMADZU CR5-A. En l'absence d'informations précises sur la nature chimique des constituants des lixiviats, les signaux sont utilisés de manière qualitative. 


\section{RESULTATS ET DISCUSSION}

\section{Les lixiviats étudiés}

Les lixiviats proviennent d'un C.E.T. de classe 2 en activité depuis 5 ans et qui reçoit des ordures ménagères compactées. Les lixiviats générés sont entièrement collectés et pompés vers un bassin de traitement. Les prélèvements sont effectués à la station de pompage. Les analyses sur le lixiviat brut (tableau 1) montrent qu'il s'agit d'un lixiviat intermédiaire à stabilisé. II est très riche en azote ammoniacal, chlorure et sodium.

Tableau 1 Paramètres physicochimiques des lixiviats étudiés.

Table 1 Physico-chemical properties of studied leachates.

\begin{tabular}{|l|c|}
\hline Paramètres & Valeurs moyennes \\
\hline pH & 7.6 à 8 \\
$\mathrm{DCO}_{\left(\mathrm{mgO}_{2} / \mathrm{l}\right)}$ & 2500 à 3500 \\
$\mathrm{DBO}_{5}\left(\mathrm{mgO}_{2} / \mathrm{l}\right)$ & 200 à 1000 \\
Azote $\mathrm{Kjeldahl}(\mathrm{mgN} / \mathrm{l})$ & 1800 à 2200 \\
Azote Ammoniacal $(\mathrm{mgN} / \mathrm{l})$ & 1600 à 2000 \\
Fer total $(\mathrm{mgFe} / \mathrm{l})$ & 14 \\
Matières en suspension $(\mathrm{mg} / \mathrm{l})$ & 45 \\
Rapport $\mathrm{DBO}_{5} / \mathrm{DCO}$ & 0,1 à 0,3 \\
Sodium $(\mathrm{mg} / \mathrm{l})$ & 1200 à 2500 \\
Calcium $(\mathrm{mg} / \mathrm{l})$ & 100 a 300 \\
Magnésium (mg/l) & 100 à 150 \\
Chlorures (mg/l) & 1500 à 3000 \\
Sulfates (mg/l) & 40 à 70 \\
\hline
\end{tabular}

Le recouvrement de la matière organique par ultrafiltration est vérifié sur six lixiviats prélevés au cours de l'année 1996. Pour chacun d'entre eux nous avons constaté que la somme des DCO de chaque fraction était égale à la DCO du lixiviat brut filtré. En moyenne, la fraction $\mathrm{F} 1$ représente $35 \%$ de la DCO, F2 $11 \%, \mathrm{~F} 313 \%$ et $\mathrm{F} 441 \%$. La fraction F4 renferme de 70 à $80 \%$ de la partie minérale du lixiviat.

L'étude chromatographique a été réalisée sur un échantillon prélevé en janvier 1997 (ultrafiltration dès le prélèvement puis congélation des fractions en attente des analyses). Nous avons vérifié la reproductibilité des profils chromatographiques par l'analyse de deux autres échantillons prélevés en mai et octobre 1996.

\section{Les conditions chromatographiques}

\section{Les éluants}

Le gel TSK-PW est un polymère hydrophile contenant le groupement polyéther hydroxylé $-\mathrm{CH}_{2} \mathrm{CHOH} \mathrm{CH} \mathrm{H}_{2} \mathrm{O}$-. Nous avons choisi ce gel semi-rigide car il est caractérisé par une faible adsorption des polysaccharides, des polymères 
solubles en milieux aqueux et par une grande stabilité sur une gamme de $\mathrm{pH}$ de 2 à 12 (HASHIMOTO et al., 1978). Diverses études montrent toutefois que des phénomènes autres que ceux régissant strictement l'exclusion stérique interviennent et nécessitent l'ajout de modificateurs dans le solvant d'élution (DUBIN et al., 1981 ; DUBIN et al., 1982 ; KATO et al., 1980). Des phénomènes d'adsorption de substances cationiques et d'exclusion de composés anioniques sont observés. Ils sont dus aux nombreux groupements hydroxyles, mais aussi à une petite quantité de groupements carboxyles présents sur le gel. Ces phénomènes peuvent être réduits en employant des éluants capables de supprimer ou de masquer l'ionisation des groupements parasites; des solutions salines ont souvent été proposées ainsi que de l'eau acidifiée (KATO et al., 1980 ; KATO, 1989). Les contraintes du DEDL nécessitant l'utilisation d'éluants évaporables nous ont amené à choisir l'eau acidifiée par de l'acide acétique.

Des interactions hydrophobes sur les sites apolaires du gel ont aussi été notées ; cet effet est habituellement réduit par l'ajout d'un solvant organique, acétonitrile ou méthanol (DUBIN et al., 1984 ; KATO et al., 1980). Nous utiliserons un mélange eau-méthanol (70-30), une précipitation ayant été constatée lors de l'ajout d'acétonitrile dans les lixiviats étudiés.

Les temps de rétention correspondant aux volumes d'exclusion et de perméation totales ( $\mathrm{Ve}$ et $\mathrm{Vp}$ ) des colonnes ont été déterminés respectivement avec du bleu dextran $\left(P M=2.10^{6} \mathrm{Da}\right)$ et du glucose $(P M=186 \mathrm{Da})$ en détection DEDL. Ils sont, pour un débit d'éluant de $0,5 \mathrm{ml} / \mathrm{min}$ :

- Ve : 8,7 min, Vp : 17,4 min pour la colonne G3000 ;

- Ve : 18,0 min, Vp : 37,2 min pour les colonnes G5000 et G3000 en série.

Nous n'avons pas constaté de variation de ces volumes en fonction de l'éluant utilisé.

Compte tenu, d'une part de la présence dans la fraction F4 de sels en concentration importante et d'autre part de la détection de ceux-ci par le DEDL, nous avons déterminé les temps de rétention de $\mathrm{NaCl}$ sur trois colonnes $\mathrm{G} 3000$ (tableau 2). On note une augmentation du temps de rétention en fonction de la concentration. Un tel comportement a aussi été observé sur gel Sephadex (DUBIN, 1988 ; HEJZLAR, 1987) où les pics d'élution des sels peuvent varier du volume d'exclusion totale pour de faibles concentrations au volume de perméation totale pour de fortes teneurs ; les effets sont dus aux forces de répulsion existant entre les anions et le gel électronégatif.

Tableau 2 Temps de rétention du chlorure de sodium en fonction de sa concentration.

Colonne : TSK PW, G3000 ; Débit de l'éluant : $0,5 \mathrm{ml} / \mathrm{min}$; Détection : DEDL

Table 2 Effect of sodium chloride concentration on retention time. Column: TSK-PW, G3000; Flow rate: $0,5 \mathrm{ml} / \mathrm{min}$; Detection: ELSD

\begin{tabular}{|c|c|c|c|c|c|c|c|c|c|c|c|c|}
\hline & \multicolumn{3}{|c|}{$\begin{array}{c}\text { Eau-méthanol } \\
\text { 70-30 } \\
\text { Colonne 1 }\end{array}$} & \multicolumn{4}{c|}{$\begin{array}{c}\text { Eau pH 4 } \\
\text { Colonne 2 }\end{array}$} & \multicolumn{3}{|c|}{$\begin{array}{c}\text { Eau pH 4 } \\
\text { Colonne 3 }\end{array}$} \\
\hline $\mathrm{Na} \mathrm{Cl}(\mathrm{g} / \mathrm{l})$ & 0,05 & 0,1 & $\mathbf{0 , 5}$ & $\mathbf{1}$ & 0,05 & $\mathbf{0 , 1}$ & 0,5 & 1 & 0,05 & 0,1 & 0,5 & 1 \\
$\mathrm{TR}(\mathrm{min})$ & 13,4 & 14,8 & 17,8 & 18,2 & 12,6 & 13,6 & 15,6 & 17,8 & 16,4 & 17,6 & 19,2 & 19,4 \\
\hline
\end{tabular}


En éluant eau $\mathrm{pH} 4$, la répulsion du sel est moins forte sur la colonne 3 que sur la colonne 2 : la charge électronégative de la colonne 3 est sans doute moindre que celle de la colonne 2.

\section{Les délecteurs}

L'utilisation des colonnes dans les conditions décrites ci-dessus conduit généralement à des amas chromatographiques qu'il est possible de résoudre partiellement en pics ou amas secondaires en utilisant trois détecteurs. En choisissant les longueurs d'onde d'absorption en détection UV-visible, d'excitation et d'émission en détection fluorimétrique, on peut mettre en évidence certaines familles de molécules de manière préférentielle. Le détecteur DEDL ne fournit aucune information structurale, mais peut donner des indications semi-quantitatives.

En effet, si au plan quantitatif, les lois expérimentales des trois détecteurs relient l'intensité du signal à la quantité de soluté, elles sont cependant la traduction de phénomènes différents et chaque loi a donc une forme spécifique. Dans l'expression de ces lois, des coefficients de proportionnalité (rendement de fluorescence, coefficient d'extinction en absorption UV-visible, exposant massique en diffusion de lumière) dépendent de la nature des molécules de façon plus ou moins importante. Ceci vaut surtout pour les techniques UVVisible et fluorimétrique qui seront seulement utilisées comme outil de caractérisation et d'aide à la résolution des pics chromatographiques. Le détecteur DEDL est en revanche utilisable pour n'importe quel soluté et l'intensité du signal dépend beaucoup moins de la nature chimique des molécules ; en première approche, nous admettrons que le chromatogramme obtenu en détection DEDL est un indicateur de l'abondance relative des molécules ou familles de molécules correspondant à chaque pic ou amas chromatographique.

Les longueurs d'onde de travail : $254 \mathrm{~nm}$ en UV (composés aromatiques), $320 / 430$ et $275 / 325$ (Exc. $\mathrm{nm} / \mathrm{Em}$. $\mathrm{nm}$ ) en fluorimétrie, ont été choisies par référence à des travaux antérieurs (BELIN et al., 1993 ; COBLE, 1996 ; GOURDON et al., 1989 ; MEJBRI et al., 1995 ; MOPPER et al., 1993). Pour l'étude fluorimétrique, elles sont caractéristiques, selon ces auteurs, respectivement des substances type-humique et type-protéine.

\section{La fraction F4 .}

Pour l'analyse de cette fraction, nous avons utilisé trois colonnes G3000, l'une pour l'éluant eau-méthanol (col.1), les deux autres pour l'eau acidifiée (col.2 et col.3).

En élution eau-méthanol (figure 2) l'observation des profils chromatographiques obtenus avec les quatre détections montre une séparation en familles de composés présentant des caractéristiques différentes . Le pic à 9,5 min, peu intense en détection DEDL, devient majoritaire en UV à $254 \mathrm{~nm}$ et en fluorimétrie dans les conditions $320 / 430 \mathrm{~nm}$. En fluorimétrie à $275 / 325 \mathrm{~nm}$ (type-protéine) un autre pic devient majoritaire vers $18 \mathrm{~min}$; les molécules fluoresçant dans ces conditions sont incluses dans l'amas observé en diffusion de lumière entre 15 et $20 \mathrm{~min}$. L'intensité importante de cet amas nous a conduit à rechercher d'autres composés. Nous avons analysé les sucres (méthode à l'anthrone sulfurique) ; la faible teneur mesurée ( $40 \mathrm{mg} / \mathrm{L}$ en équivalent glucose) n'explique pas l'importance du signal DEDL. 


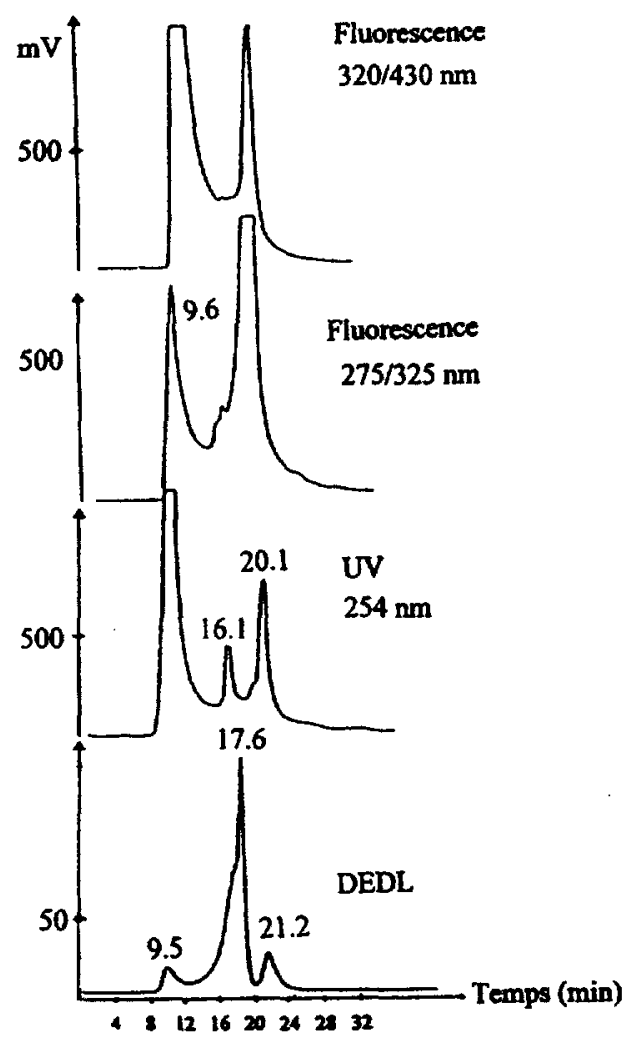

Figure 2 Chromatogrammes de perméation de gel de la fraction F4.

Colonne : TSK PW, G3000 ; Eluant : eau-méthanol, $70-30$; Débit : $0,5 \mathrm{ml} / \mathrm{min}$

SEC chromatograms of fraction F4.

Column: TSK PW, G3000; Mobile phase: mixture water-methanol, 70-30; Flow rate: $0,5 \mathrm{ml} / \mathrm{min}$

En élution eau $\mathrm{pH} 4$, les profils obtenus en détection DEDL sur les colonnes 2 et 3 sont différents (figure 3). La force ionique de l'éluant eau pH 4 n'est sans doute pas suffisante pour supprimer les effets électrostatiques perturbant l'exclusion stérique. Cependant, le chromatogramme obtenu à l'aide de la colonne 3 en eau pH 4 est comparable à celui observé en élution eau-méthanol sur la colonne 1. Ils ne diffèrent que par la présence du pic à $9.5 \mathrm{~min}$, observé en éluant eau - méthanol, traduisant un phénomène d'exclusion ionique, fortement atténué en éluant eau $\mathrm{pH} 4$. II sera intéressant de mettre à profit ces interactions secondaires pour améljorer le fractionnement et la caractérisation de F4. 


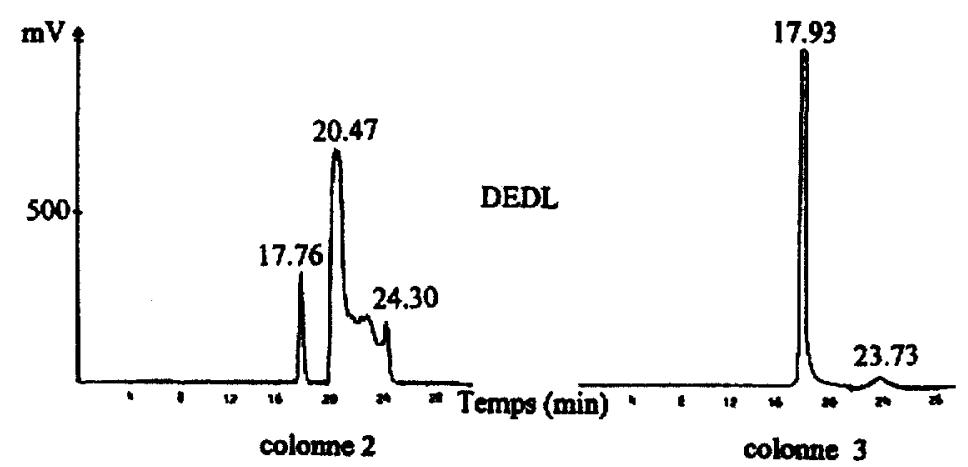

Figure 3 Chromatogrammes de perméation de gel de la fraction F4. Colonnes : TSK PW, G3000 ; Eluant : Eau pH 4 ; Débit : $0,5 \mathrm{ml} / \mathrm{min}$

SEC chromatograms of fraction F4.

Columns: TSK PW, G3000; Mobile phase: water at pH 4 with acetic acid; Flow rate: $0,5 \mathrm{~m} / \mathrm{min}$

\section{Les fractions F1, F2 et F3}

La chromatographie de perméation de gel sur Sephadex et des études IR et RMN réalisées sur des lixiviats ont permis à plusieurs auteurs de comparer la matière organique de ces effluents aux substances humiques naturelles. Dans ces substances, alors que les acides fulviques sont solubles à la fois en solutions acide et alcaline, les acides humiques précipitent en milieu acide (POWELL et al., 1992 ; SCHNITZER, 1991). La précipitation à pH 1 permet d'ailleurs le fractionnement de ces substances dans les eaux naturelles (THURMAN et al., 1981) et dans les lixiviats (MEJBRI et al., 1995). Pour les fractions F1, F2 et F3, nous avons constaté une précipitation après acidification. Aussi, afin de ne pas modifier leurs compositions par l'acidification, nous avons réalisé l'étude chromatographique uniquement en élution eau-méthanol.

La figure 4 présente les chromatogrammes obtenus en détection DEDL. On observe de F4 à F1, l'apparition progressive de produits plus lourds.

La comparaison des chromatogrammes en détection DEDL, UV et fluorimétrie est présentée sur la figure 5 pour les fractions F3 et F2 et sur la figure 6 pour la fraction F1. L'analyse de cette fraction est réalisée sur les colonnes G5000 et G3000 en série afin de mieux séparer les composés lourds.

Les fractions $\mathrm{F} 3$ et $\mathrm{F} 2$ ont des profils voisins ; pour F3 les temps de rétention sont légèrement supérieurs à ceux de F2. L'intensité des signaux observés en détection DEDL est faible en particulier pour les pics aux temps de rétention 15 et $23 \mathrm{~min}$. Seule la présence du pic vers 9 min mérite d'être notée compte tenu de l'importante intensité de fluorescence à $320 / 430 \mathrm{~nm}$. II correspond à des substances type-humique.

La fraction F1 est caractérisée par un massif DEDL important (de 14 à $22 \mathrm{~min}$ ) . Deux groupes de composés s'interpénètrent : ceux éluant vers 20 min qui absorbent en UV à $254 \mathrm{~nm}$ et fluorescent à $320 / 430 \mathrm{~nm}$, et ceux vers $16 \mathrm{~min}$, temps de rétention proche du volume d'exclusion totale traduisant la 


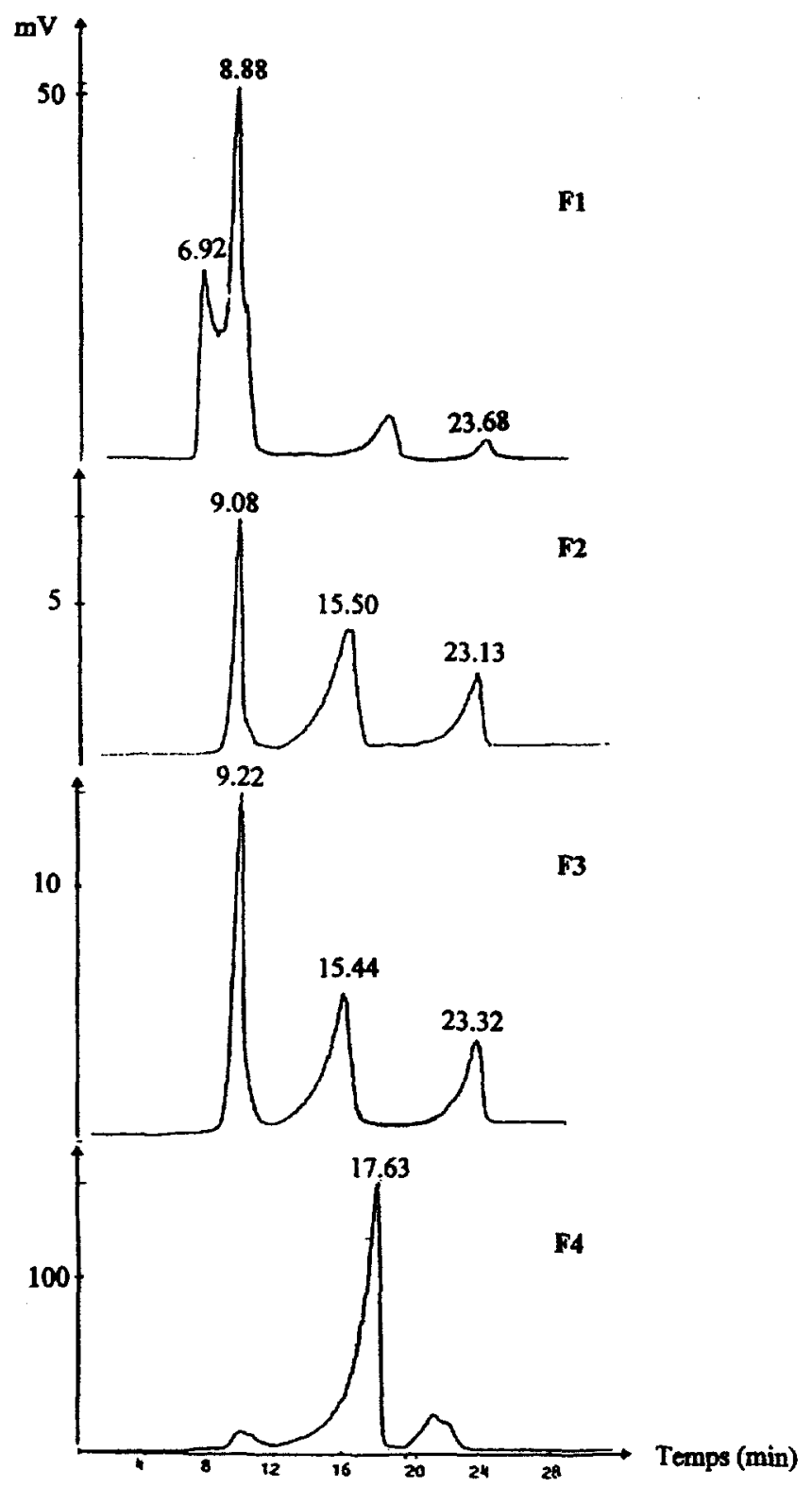

Figure 4 Chromatogrammes de perméation de gel des fractions F1, F2, F3 et F4.

Colonne TSK PW, G3000 ; Détection : DEDL ; Eluant : eau-méthanol, 70-30 : Débit : $0.5 \mathrm{~mL} / \mathrm{min}$

SEC chromatograms of fractions $F 1, F 2, F 3$ and $F 4$.

Column: TSK PW, G3000; Detection: ELSD; Mobile phase: mixture water-methanol, 70-30: Flow rate: $0,5 \mathrm{ml} / \mathrm{min}$ 


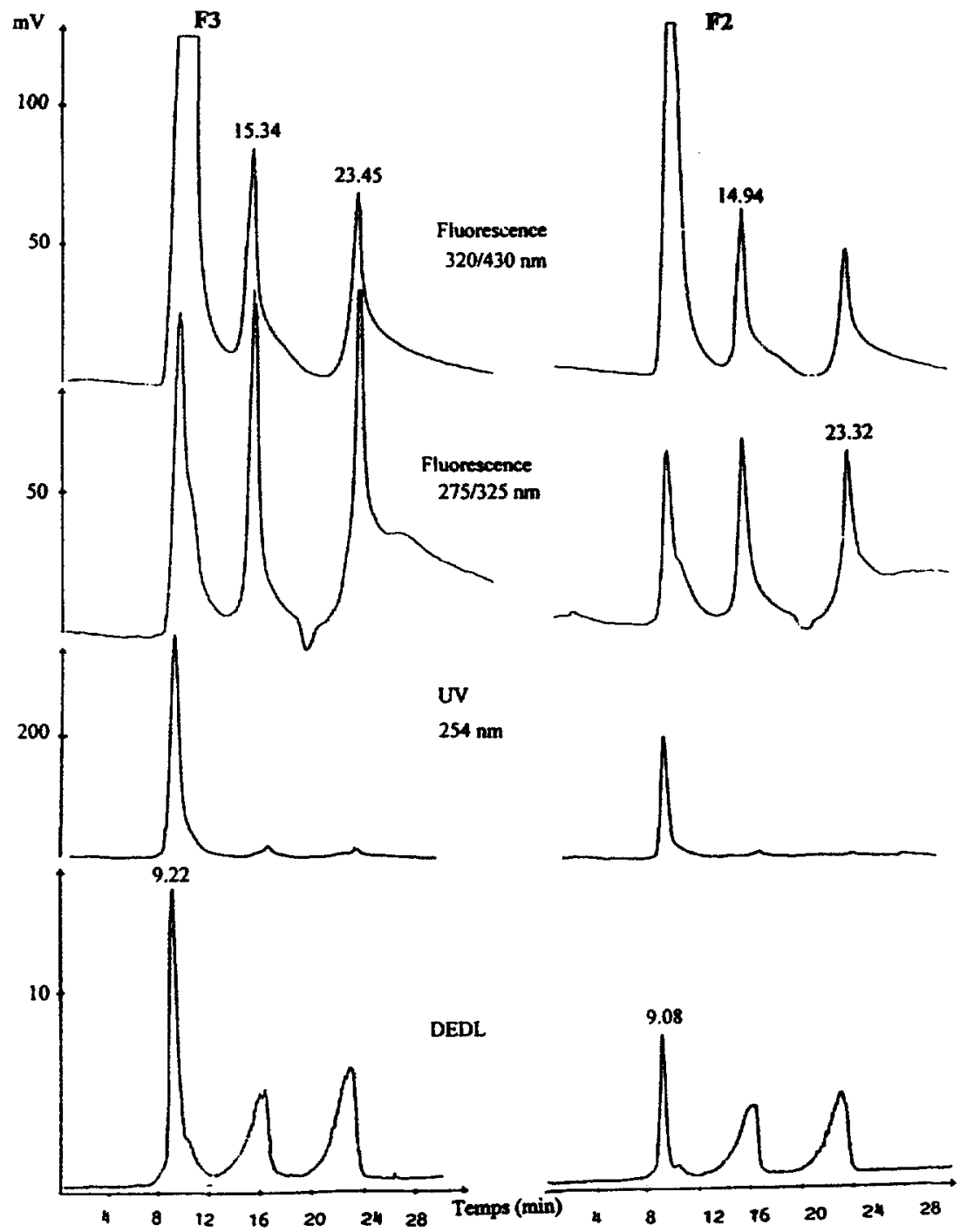

Figure 5 Chromatogrammes de perméation de gel des fractions F2 et F3. Colonne : TSK PW, G3000 ; Eluant : eau-méthanol, $70-30$; Débit : $0,5 \mathrm{ml} / \mathrm{min}$ SEC chromatograms of fractions F2 and F3.

Column: TSK PW, G3000; Mobile phase: mixture water-methanol, 70-30; Flow rate: $0,5 \mathrm{ml} / \mathrm{min}$

présence de composés à hauts poids moléculaires. Ils absorbent peu à $254 \mathrm{~nm}$, mais présentent un signal très intense en fluorimétrie à $275 / 325 \mathrm{~nm}$.

En résumé, dans les fractions $F 3, F 2$ et F1, nous notons la présence de produits de poids moléculaires apparents élevés. Nous distinguons deux familles 


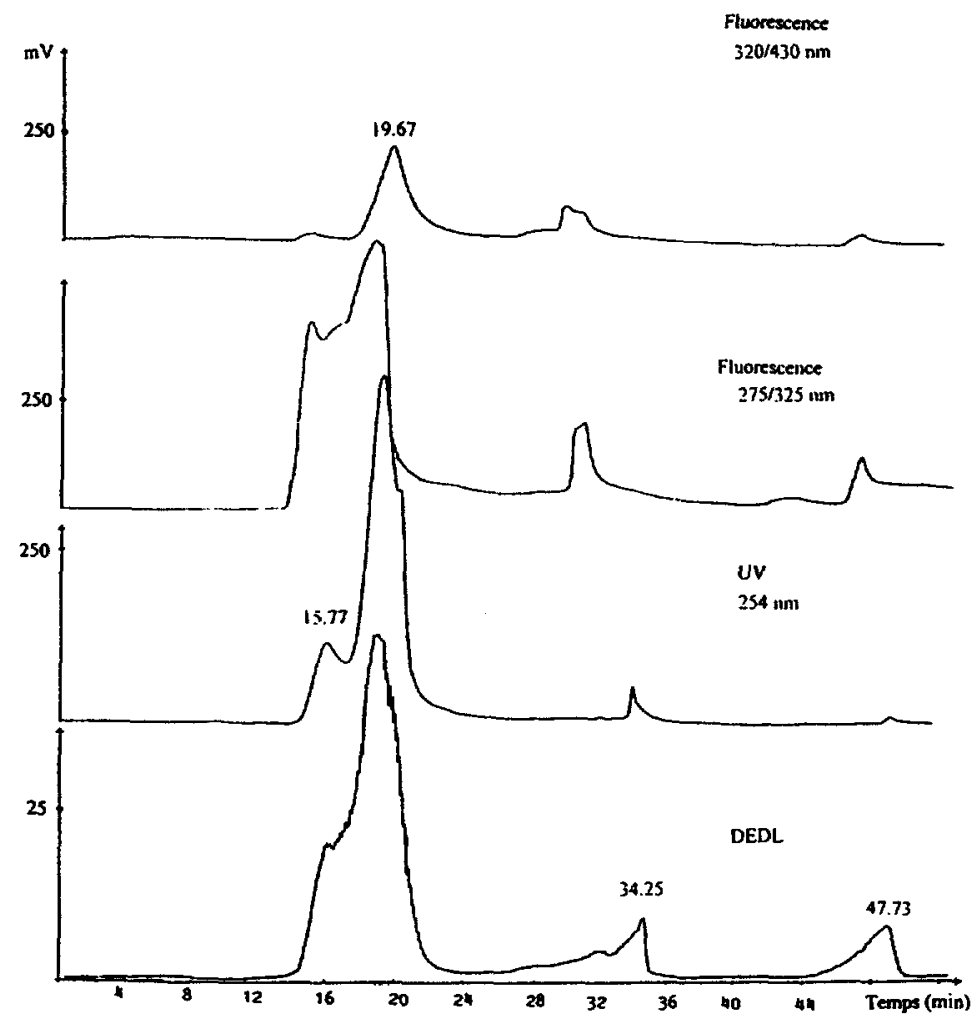

Figure 6 Chromatogrammes de perméation de gel de la fraction F1.

Colonnes : TSK PW, G5000 et G3000 en série ; Eluant : eau-méthanol, 70-30 ; Débit : $0,5 \mathrm{ml} / \mathrm{min}$

SEC chromatograms of fraction $F 1$.

Columns: TSK PW, G5000 and G3000; Mobile phase: mixture water-methanol, 70 30; Flow rate: $0,5 \mathrm{ml} / \mathrm{min}$

de produits . L'une, présente dans les trois fractions, est caractérisée par une fluorescence dans les conditions d'excitation et d'émission des substances humiques. La seconde famille, présente dans F1 seulement, fluoresce avec une forte intensité dans les conditions des substances protéinées. Nous avons confirmé la présence des protéines par un dosage des acides aminés d'après la méthode décrite par DOSSIER BERNE et al. (1994); dans cette fraction, la somme des acides aminés dosés représente $93 \%$ de l'azote organique alors qu'elle n'est que de 3 à $5 \%$ pour les autres fractions. 


\section{CONCLUSION}

Cette étude préliminaire a permis d'évaluer l'intérêt de la chromatographie haute performance sur gel d'exclusion stérique pour caractériser la matière dissoute de lixiviats de centres d'enfouissement technique de déchets ménagers. En terme de poids moléculaires, il y a surtout deux groupes de molécules :

- un groupe de petites molécules (PMA $<1000$ Da) qui sont éluées à un volume proche du volume de perméation totale. Des composés type-humique et type-protéine sont présents dans ce groupe mais également des molécules de nature autre, que nous n'avons pu identifier. L'élution de ces molécules est fortement perturbée par des interactions secondaires ;

- un groupe de molécules de PMA > $10000 \mathrm{Da}$; pour ce groupe deux familles de molécules sont mises en évidence en comparant les résultats obtenus à l'aide de trois détecteurs. L'une est composée de produits de typehumique, l'autre de protéines.

Les produits de poids moléculaires intermédiaires sont apparemment peu nombreux.

À ce stade notre étude n'a pas permis d'aller au-delà de ces observations, mais elle ouvre des perspectives intéressantes. La combinaison chromatographie d'exclusion stérique - multidétection est prometteuse ; une étude spectrométrique approfondie est actuellement en cours pour déterminer les conditions de détection spécifiques à chaque groupe de composés. Les caractéristiques spectrales ainsi définies seront utilisées pour affiner la séparation chromatographique des lixiviats; les interactions secondaires seront mises à profit.

\section{RÉFÉRENCES BIBLIOGRAPHIQUES}

BELIN C., QUELLEC C., LAMOTTE M., EWALD M., SIMON Ph., 1993. Characterization by fluorescence of the dissolved organic matter in natural water. Application to fractions obtained by tangential ultrafiltration and XAD resin isolation. Environ. Technol., 14, 1131-1144.

CHAISSAC M., AUDIC J.M., FAUP G.M., 1987. Un outil analytique de caractérisation des eaux résiduaires : la chromatographie de filtration sur gel. T.S.M., 9, 389-395.

CHIAN E.S.K., DE WALLE F.B., 1977. Characterization of soluble organic matter in leachate. Environ. Sci. Technol., 11(2), 158-163.

CLEMENT B., THOMAS O., 1994. Application of ultra-violet spectrophotometry and gel permeation chromatography to the cha- racterization of landfill leachates. Environ. Technol., 16, 367-377.

COBLE P.G., 1996. Characterization of marine and terrestrial DOM in seawater using excitation - emission matrix spectroscopy. Marine chemistry, 51, 325-346.

DE HAAN H., DE BOER T., 1978. A study of the possible interactions between fulvic acids, amino acids and carbohydrates from Tjeukemeer, based on gel filtration at pH 7, Water Research, 12, 1035-1040.

DOSSIER BERNE F., PANAIS B., MERLET N., CAUCHI B., LEGUBE B., 1994. Analyse des acides aminés dissous totaux dans les eaux à potabiliser en cours de traitement. Environ. Technol., 15, 901-916.

DREUX M., LAFOSSE M., MORINALLORY L., 1996. The Evaporative Light 
Scattering Detector: A universal instrument for non volatile solutes in LC and SFC. LC.GC International, March.

DUBIN P.L., LEVY I.J., 1981. High performance aqueous exclusion chromatography of polycations on a hydrophilic gel column. Polym. Preprints, 22(1), 132-134.

DUBIN P.L., LEVY I.J., 1982. Gel permeation chromatography of cationic polymers on PW gel columns. J. Chromatogr., 235, 377-387.

DUBIN P.L., LEVY I.J., OTERI R., 1984. Hydrophobic effects in exclusion chromatography on PW gel columns. J. Chromatogr., 22, 432-434.

DUBIN P.L., 1988. Aqueous size-exclusion chromatography. J Chromatography library, $453 p$.

FORST C., STIEGLITZ L., ROTH W., KUHNMUNCH S., 1989. Quantitative analysis of volatile organic compounds in landfill leachates. Inter. J. Environ. Anal. Chem., 37, 287-293.

GOURDON R., COMEL C., VERMANDE P., VERON J., 1989. Fractionation of the organic matter of a landfill leachate before and after aerobic or anaerobic biological treatment. Water Research, 23(2), 167-173.

GRANET C., MILLOT N., WICKER A., NAVARRO A., VERON J., 1985. Application de la chromatographie de permeation sur gel aux lixiviats de décharge contrôlée. T.S.M., 5, 223-229.

HARMSEN J., 1983. Identification of organic compounds in leachate from a waste tip. Water Research, 17(6), 699-705.

HASHIMOTO T., SASAKI H., AIURA M., KATO Y., 1978. High-speed aqueous gel permeation chromatography. J. Polymer Science, 16, 1789-1800.

HEJZLAR J., 1987. Effect of inorganic salts and adsorption in Sephadex gel chromatography of aquatic organic substances. Water Research, 21(11), 1311-1318.

HONGVE D., BAANN J., BECHER G., 1996. Characterization of humic substances by means of high performance size exclusion chromatography. Environ. International, 22(5), 489-494.

KATO Y., KOMIYA K., SASAKI H., HASHIMOTO T., 1980. Comparaison of TSKGEL PW type and SW type in high-speed aqueous gel permeation chromatography. J. Chromatogr., 193, 311-315.
KATO Y., 1989. Size exclusion chromatography. Edited by HUNT, B.J. and HOLDING S.R.

MANNI G., CARON F., 1995. Calibration and determination of volatile fatty acids in waste leachates by gas chromatography. J. Chromatogr. A, 690, 237-242.

MATSUBARA H., URANO K., 1994. Measurement of molecular weight distribution of humic substances with untreated Sephadex G-15 and ammonia-treated Sephadex G-15. Chemosphere, 29(3), 485-494.

MEJBRI R., MATEJKA G., LAFRANCE P., MAZET M., 1995. Fractionnement et caractérisation de la matière organique des lixiviats de décharges d'ordures ménagères. Revue des Sciences de l'eau, 8, 217-236.

MERTENS P., RICHELLE B., WATELET M., WOLLAST A., 1995. Audit technique de caractérisation d'une ancienne décharge : composition d'un lixiviat particulier. T.S.M., 6, 465-470.

MILLOT N., 1986. Les lixiviats de décharge contrôlée. Caractérisation analytique. Etude des filières de traitement. Th. Doct. INSA LYON.

MILLOT N., GRANET C., WICKER A., FAUP G.M., NAVARRO A., 1987. Application of G.P.C. processing system to landfill leachates. Water Research, 21(6), 709-715.

MOPPER K., SCHULTZ C.A., 1993. Fluorescence as a possible tool for studying the nature and water column distribution of DOC components. Marine chemistry, 41, 229-238.

MURRAY H.E., BECK J.N., 1990. Concentrations of synthetic organic chemicals in leachate from municipal landfill. Environ. Pollution, 67, 195-203.

OMAN C., HYNNING P.A., 1993. Identification of organic compounds in municipal landfill leachates. Environ. Pollution, 80, 265-271.

POSNER A., 1963. Importance of electrolyte in the determination of molecular weights by “ Sephadex " gel filtration, with especial reference to humic acid. Nature, 198, 1161-1163.

POWELL H.K.J., TOWN R. M., 1992. Solubility and fractionaction of humic acid; effect of $\mathrm{pH}$ and ionic medium. Analytica chimica acta, 267, 47-54. 
REINHARD M., GOODMAN N.L., 1984. Occurrence and distribution of organic chemicals in two landfill leachate plumes. Environ. Sci. Technol., 18, 953-961.

RINAUDO M., TINLAND 8., 1991. Some problems in aqueous size exclusion chromatography of synthetic polymer and biopolymer characterization. J. of Applied Polymer Sci.: Applied Polymer Symposium, 48, 19-31.

SAWHNEY B.L., KOZLOSKI R.P., 1984. Organic pollutants in leachates from landfill sites. J. Environ. Qual., 13(3), 349-352.

SCHULTZ B., KJELDSEN P., 1986. Screening of organic matter in leachates from sanitary landfill using gas chromatography combined with mass spectrometry. Water Research, 20(8), 965-970.
SCHNITZER M., 1991. Soil organic matter The next 75 years. Soil Science, 151(1), 41-58.

SLATER C.S., UCHRIN C., AHLERT R.C., 1985. Ultrafiltration processes for the characterization and separation of landfill leachates. J. Env. Sci. Health, A20(1), 97111.

THURMAN E.M., MALCOLM R.L., 1981. Preparative isolation of aquatic humic substances. Environ. Sci. Technol., 15(4), 463-466.

URANO K., KATAGIRI K., KAWAMOTO K., 1980. Characteristics of gel chromatography using Sephadex gel for fractionation of soluble organic pollutants. Water Research, 14, 741-745. 